\title{
Experimental Entanglement Concentration and Universal Bell-state Synthesizer
}

\author{
Yoon-Ho Kim, ${ }^{1}$ 用 Sergei P. Kulik, ${ }^{2}$, Maria V. Chekhova, ${ }^{2}$, 用 Warren P. Grice, ${ }^{1}$ and Yanhua Shih ${ }^{2}$ \\ ${ }^{1}$ Center for Engineering Science Advanced Research, Computer Science and Mathematics Division, \\ Oak Ridge National Laboratory, Oak Ridge, Tennessee 37831 \\ ${ }^{2}$ Department of Physics, University of Maryland, Baltimore County, Baltimore, Maryland 21250
}

(Submitted: April 16, 2002; Revised: October 30, 2018)

\begin{abstract}
We report a novel Bell-state synthesizer in which an interferometric entanglement concentration scheme is used. An initially mixed polarization state from type-II spontaneous parametric downconversion becomes entangled after the interferometric entanglement concentrator. This Bell-state synthesizer is universal in the sense that the output polarization state is not affected by spectral filtering, crystal thickness, and, most importantly, the choice of pump source. It is also robust against environmental disturbance and a more general state, partially mixed-partially entangled state, can be readily generated as well.
\end{abstract}

PACS numbers: 03.67.-a, 42.50.-p, 42.50.Dv

Introduction - Multi-particle quantum entanglement traditionally has been associated with many fundamental issues in quantum physics, such as, the uncertainty principle and the locality, reality and causality problems in quantum theory [1]. Recently, quantum entanglement has found its applications in metrology, communication, and information processing [2]. To successfully implement these new ideas, one must be able to generate and manipulate entangled states at will. It is, however, generally recognized that even the generation of multi-particle entanglement is not trivial. Nonetheless, a great deal of work has been carried out with entangled two-qubit quantum states, or Bell-states. These states are important not only because of their simplicity, but also because of their utility in applications, such as, quantum cryptography. In addition, entangled two-qubit states may one day serve as building blocks for the construction of states of three or more entangled qubits.

The first direct generation of an entangled two-qubit state involved photon pairs produced in the process of cw-pumped type-II spontaneous parametric downconversion (SPDC) 3, 田. Although this method is still widely used for the generation of polarization entangled states, it has its limitations. In particular, the photon pair emission times are completely random. This is a drawback in applications such as quantum teleportation, multi-photon state generation, practical quantum cryptography, etc, where knowledge of the approximate times of emission are required.

Much of the uncertainty in emission time is eliminated when the SPDC process is pumped by an ultrafast laser. Unfortunately, differences in the spectral and temporal properties of the photon pair cause the polarization entanglement to suffer with this type of pumping scheme

\footnotetext{
*Electronic address: kimy@ornl.gov

${ }^{\dagger}$ Permanent address: Department of Physics, Moscow State University, Moscow, Russia
}

[5, 6]. It is possible to "concentrate" (following the definition in Ref. [7]) the entanglement by passing the photons through narrow spectral filters, effectively retaining only the more highly entangled pairs. The entanglement concentration based on a local filtering process such as this is not desirable, however, since most of the photons are simply wasted. A number of schemes involving multiple crystals have been devised to circumvent these problems [8], although none can match the simplicity and stability of a single-crystal scheme.

In this paper, we report the experimental demonstration of general entanglement concentration scheme in a two-qubit state of type-II SPDC. Our entanglement concentration scheme does not rely on local filtering. Therefore, the degree of entanglement is not affected by the pump bandwidth, the thickness of nonlinear crystal, the bandwidth of spectral filters, etc. As a result, no photons are wasted: all qubit pairs, which are initially in a mixed state, exit the entanglement concentrator as entangled qubit pairs.

Entanglement Concentration Scheme - Consider the polarization state of the photon pair generated from a type-II BBO crystal pumped either by a cw or by a ultrafast pump laser, see Fig. 11(a). As in Ref. [3], atten-

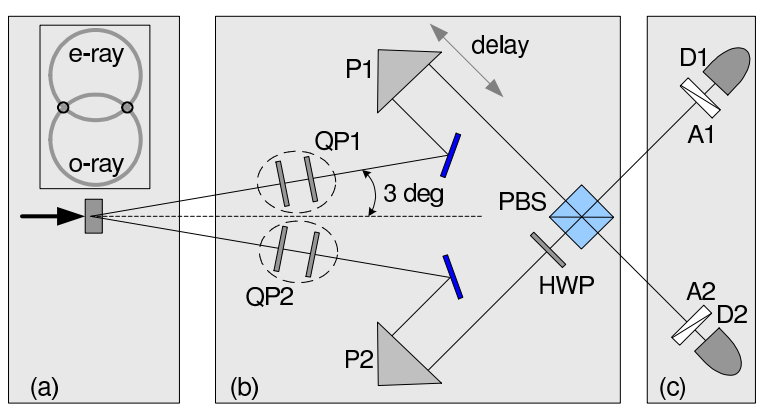

FIG. 1: (a) Non-collinear type-II SPDC is used to prepare an initial two-qubit mixed state. (b) Entanglement concentration scheme. (c) Detectors and polarization analyzers. 
(a) r-r path

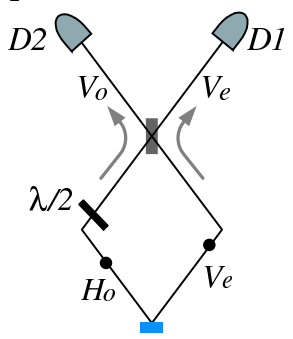

(b) t-t path

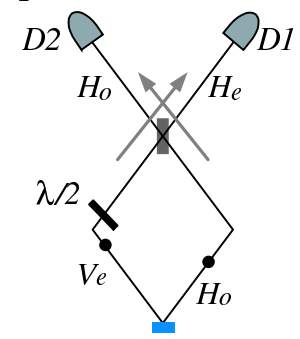

FIG. 2: Two possible quantum mechanical paths that a photon pair may follow.

tion is restricted to the intersections of the cones made by the e- and o-rays exiting the BBO crystal. In each of these two directions, a photon of either polarization (horizontal or vertical) may be found, with the orthogonal polarization found in the conjugate beam. Unlike common misconception, the photon pairs found in these two directions are not polarization entangled. In fact, the polarization state of the photon pair is in a mixed state $\rho_{\text {mix }}=\frac{1}{2}\left(\left|H_{1}\right\rangle\left|V_{2}\right\rangle\left\langle V_{2}\left|\left\langle H_{1}|+| V_{1}\right\rangle\right| H_{2}\right\rangle\left\langle H_{2}\right|\left\langle V_{1}\right|\right)$, where $|H\rangle$ and $|V\rangle$ refer to the horizontal and vertical polarization state of a single photon, respectively.

The reason the state is mixed has to do with timing information carried by the photon pair. Because of the group velocities experienced by the different polarizations are not the same, one polarization always precedes the other. Thus, the two amplitudes $\left|H_{1}\right\rangle\left|V_{2}\right\rangle$ and $\left|V_{1}\right\rangle\left|H_{2}\right\rangle$ are distinguishable in principle. In cw-pumped type-II SPDC, a pair of birefringent compensators, with the effective thickness equal to half the down-conversion crystal, can remove this timing information, thus transforming the mixed state to a pure polarization entangled state [3, 9.

But in ultrafast type-II SPDC, the pump pulse introduces additional timing information, which cannot be eliminated with the birefringent compensators, into the two-photon state [5]. It is therefore impossible to transform the mixed state into a pure polarization entangled state $[6]$.

The interferometric entanglement concentration scheme shown in Fig. 11(b), nevertheless, allows us to transform the mixed state to a polarization entangled state, regardless of the pump bandwidth, crystal thickness, and spectral filters used [10].

Let us now discuss the entanglement concentration scheme in detail, see Fig. 11(b). The photon pairs exit the crystal and travel equal distances (delay $\tau=0$ ) to a polarization beam splitter (PBS). A $\lambda / 2$ plate inserted in one arm rotates the polarization by $90^{\circ}$ and ensures that the photon pairs have the same polarization when they reach the PBS. Thus, there are two possible outcomes: both photons are reflected(r-r); or both are transmitted $(\mathrm{t}-\mathrm{t})$. These two biphoton paths are illustrated in Fig. 2 .
Since it is never the case that two photons exit the same port of the PBS, no post-selection is required. Note also that the photon that left the crystal with e-polarization (o-polarization) is always detected by $D_{1}\left(D_{2}\right)$. Therefore, the intrinsic timing information present in type-II SPDC cannot be used to distinguish between the t-t and the r-r paths. As a result, the temporal and spectral differences between the photon pair have no bearing on the polarization entanglement. We, therefore, have effectively 'dis-entangled' the timing information and the polarization information of the qubit pair. Such disentanglement of decoherence causing and the information carrying parts of the wavefunction of the qubit pair is the hallmark of this entanglement concentration scheme. (Note that the photon pair arrival times are still known within the coherence time of the pump pulse.)

The resulting amplitudes $\left|H_{1}\right\rangle\left|H_{2}\right\rangle$ and $\left|V_{1}\right\rangle\left|V_{2}\right\rangle$ are then quantum mechanically indistinguishable. Therefore, they are in quantum superposition $|\Phi\rangle=$ $\frac{1}{\sqrt{2}}\left(\left|H_{1}\right\rangle\left|H_{2}\right\rangle+e^{i \varphi}\left|V_{1}\right\rangle\left|V_{2}\right\rangle\right)$, where $\varphi$ is the phase between the two term and it may be varied by tilting the phase plates QP1 and QP2. With a setting of $\varphi=0$, the density matrix of the output state can be written as $\rho_{\text {ent }}=\left|\Phi^{(+)}\right\rangle\left\langle\Phi^{(+)}\right|$.

If the delay $\tau$ is introduced in one arm relative to the other, then the overall overlap between the two amplitudes becomes smaller. In this case, a more general state, partially mixed-partially entangled state, $\rho=$ $\varepsilon \rho_{\text {ent }}+(1-\varepsilon) \rho_{\text {mix }}$, where $0 \leq \varepsilon \leq 1$, is generated.

Universal Bell-state Synthesizer - To understand why this entanglement concentration scheme works as a universal Bell-state synthesizer, it is necessary to carry out fully quantum mechanical calculation of the joint detection rate. What we would like to show here is that the quantum interference at $\tau=0$ (balanced interferometer) does not depend on any parameters that are related to the pump source, spectral filtering, and the crystal properties. We present a brief summary of the calculation here and a detailed calculation will be published elsewhere [11].

The coincidence counts $R_{c}$ is proportional to

$$
R_{c} \propto \int d t_{1} d t_{2}\left|\left\langle 0\left|E_{1}^{(+)}\left(t_{1}, \tau\right) E_{2}^{(+)}\left(t_{2}, \tau\right)\right| \psi\right\rangle\right|^{2}
$$

where $|\psi\rangle$ is the state of type-II SPDC [5]. Assuming that the quartz phase plates are adjusted so that $\varphi=0$, the electric field operators that reach the detectors in this experiment can be written as

$$
\begin{aligned}
E_{1}^{(+)}\left(t_{1}, \tau\right)= & \int d \omega^{\prime}\left\{\cos \theta_{1} e^{-i \omega^{\prime}\left(t_{1}+\tau\right)} a_{V e}\left(\omega^{\prime}\right)\right. \\
& \left.-\sin \theta_{1} e^{-i \omega^{\prime} t_{1}} a_{H e}\left(\omega^{\prime}\right)\right\} \\
E_{2}^{(+)}\left(t_{2}, \tau\right)= & \int d \omega^{\prime}\left\{\cos \theta_{2} e^{-i \omega^{\prime} t_{2}} a_{V o}\left(\omega^{\prime}\right)\right. \\
& \left.-\sin \theta_{2} e^{-i \omega^{\prime}\left(t_{2}+\tau\right)} a_{H o}\left(\omega^{\prime}\right)\right\}
\end{aligned}
$$




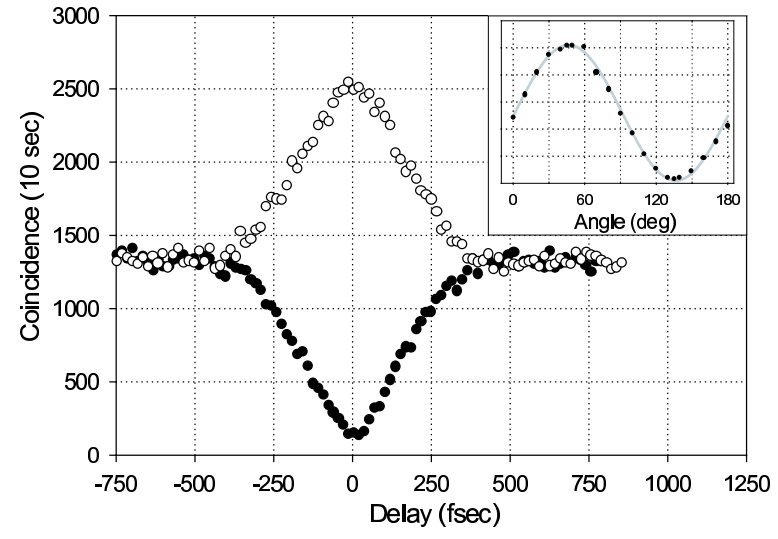

FIG. 3: Experimental data for cw-pumped type-II SPDC. $\varphi=0$ for open circle $\left(\left|\Phi^{(+)}\right\rangle\right)$and $\varphi=\pi$ for solid circle $\left(\left|\Phi^{(-)}\right\rangle\right)$. Inset shows the polarization interference for $\left|\Phi^{(+)}\right\rangle$ state.

where, for example, $a_{V o}\left(\omega^{\prime}\right)$ is the annihilation operator for a photon of frequency $\omega^{\prime}$ with vertical polarization which was originally created as the o-ray of the crystal. $\theta_{1}$ and $\theta_{2}$ are the angles of the polarization analyzers $A_{1}$ and $A_{2}$, respectively.

Upon carrying out the calculation, we find that the coincidence count rate has the form

$$
\begin{array}{r}
R_{c} \propto \int d t_{+} d t_{-} \mid \cos \theta_{1} \cos \theta_{2} \Pi\left(t_{+}+\tau / 2, t_{-}+\tau\right) \\
+\left.\sin \theta_{1} \sin \theta_{2} \Pi\left(t_{+}+\tau / 2, t_{-}-\tau\right)\right|^{2} .
\end{array}
$$

The detailed expression of $\Pi\left(t_{+}, t_{-}\right)$is given in Ref. 88, 11 and it contains all the important factors such as crystal length, pump bandwidth, bandwidth of spectral filters, etc.

Let us now look at Eq. (11) carefully. If the interferometer is balanced, i.e. $\tau=0, \Pi\left(t_{+}, t_{-}\right)$factors out and becomes a constant. Therefore, $R_{c} \propto \cos \left(\theta_{1}-\theta_{2}\right)^{2}$, a clear signature of polarization entangled state $\left|\Phi^{(+)}\right\rangle$. This means that, when $\tau=0$, all parameters that affect the temporal shape of the $\Pi\left(t_{+}, t_{-}\right)$, including the pump bandwidth, the crystal properties, the crystal thickness, and the filter bandwidth, simply do not have any effect on the quantum interference. Due to this reason, this scheme can be considered as a universal Bell-state synthesizer.

Experiment - As described above, the initial polarization state, a mixed state, was prepared by type-II non-collinear frequency-degenerate SPDC. A $3 \mathrm{~mm}$ BBO crystal was pumped by a cw argon ion laser operating at $351.1 \mathrm{~nm}$, producing photons with a central wavelength of $702.2 \mathrm{~nm}$. The delay $\tau$ was introduced through the motion of one of the trombone prisms and the phase between the two alternatives (shown in Fig. 2) was adjusted by slightly tilting the $600 \mu \mathrm{m}$ quartz plates QP1 in opposite directions. The optic axes of the quartz crystals were oriented vertically. The outputs from the two de-

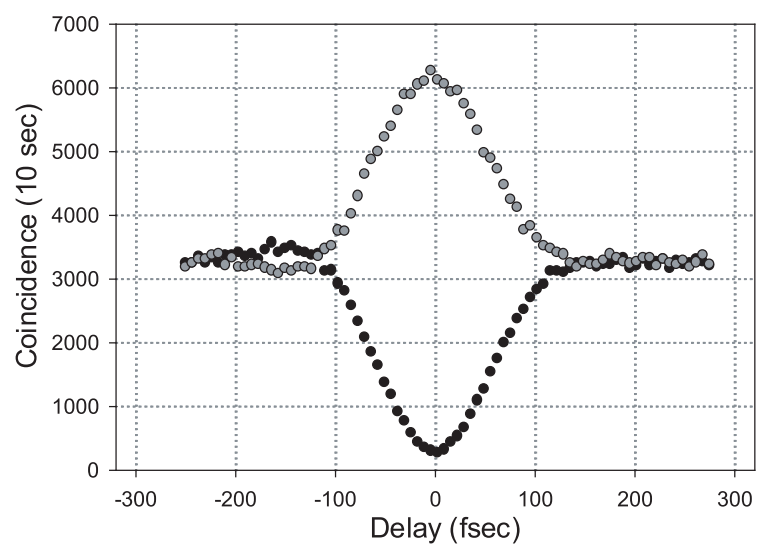

FIG. 4: Experimental data for ultrafast type-II SPDC. Analyzer angles were $45^{\circ} / 45^{\circ}$ for the peak and $45^{\circ} /-45^{\circ}$ for the dip.

tectors were fed to a time-to-amplitude converter (TAC) and the TAC output was analyzed by a multi-channel analyzer with a coincidence window set to 3 nsec. No spectral filters were used in this experiment.

First, we have done typical space-time interference experiments by varying the delay $\tau$ with both polarization analyzers set at $45^{\circ}$. The phase term was adjusted by tilting the quartz plates $\mathrm{QP} 1$ and $\varphi=0$ and $\varphi=\pi$ were selected to prepare Bell-states $\left|\Phi^{(+)}\right\rangle$and $\left|\Phi^{(-)}\right\rangle$, respectively. Fig. 3 shows the experimental data. When the delay $\tau$ is zero, i.e., no path length difference between the two arm, complete destructive or constructive interference is observed. Note that this is different from that of typical type-II case in which $\tau$ equal to half the crystal thickness should be inserted to observe complete quantum interference [3, 9]. The typical triangular twophoton wavepacket is clearly demonstrated and the base width of the triangular wavepacket agrees well with the theoretically expected value of $742 \mathrm{fsec}$ 9, 11].

The inset of Fig. 3 shows the polarization interference for $\left|\Phi^{(+)}\right\rangle$state. $A_{1}$ was fixed at $45^{\circ}$ and $A_{2}$ was rotated. Expected $\cos \left(\theta_{1}-\theta_{2}\right)^{2}$ correlation is clearly demonstrated.

We have repeated the above space-time and polarization measurements with several different spectral filters and found almost no change in the quality of quantum interference. The stability of the interferometer is also checked by repeating the polarization interference measurement several times at different times and we have found almost no change in the visibility of the polarization interference. This is basically due to the fact that a small change around $\tau=0$ causes very little effects on the quantum interference as shown in Fig. 3 .

We have also done the experiment in which an ultrafast laser is used as the pump. A second harmonic (390 $\mathrm{nm}$ ) of a commercial mode-locked Ti:Sa laser operating at $780 \mathrm{~nm}$ was used as a pump. The bandwidth of the 
pump pulse was about $2 \mathrm{~nm}$. The thickness of the type-II BBO crystal was $3 \mathrm{~mm}$ and the SPDC radiation was centered at $780 \mathrm{~nm}$. Interference filters with $20 \mathrm{~nm}$ FWHM were inserted in front of each detectors to reduce noise counts. The effective coincidence window for this experiment was 3 nsec. The quartz phase plates QP1 and QP2 were not available for this experiment so the phase $\varphi$ could only be roughly adjusted by the tilt angle of the crystal itself. Since the phase $\varphi$ could not be freely adjusted, we fixed $\varphi$ at some arbitrary angle and scanned the delay $\tau$ for analyzer angles $45^{\circ} / 45^{\circ}$ for the peak and $45^{\circ} /-45^{\circ}$ for the dip. Fig. 目 shows a typical data set for this measurement. The observed visibility is greater than $90 \%$ and could have been improved further if phase plates had been available to fine tune $\varphi$. Nevertheless, the observed visibility is by far the highest in ultrafast type-II SPDC generated from a thick crystal (For comparison, see Ref. [6] for usual ultrafast type-II SPDC data) and in principle it does not depend on crystal, pump, and filter parameters as shown in Eq. (1). The FWHM of the interference is roughly estimated to be $140 \sim 150$ fsec which is very close to theoretically expected value of 160 fsec (assuming exactly $2 \mathrm{~nm}$ FWHM pump bandwidth). [1].

Discussion - It is helpful to introduce the terms "Entanglement" or "Entanglement of Formation" $(E)$ and "Entropy" or "Entropy of Entanglement" $(S)$ formally, as done in Ref. [7], to visualize the entanglement concentration process in this work.

Under these definitions, our initial mixed two-qubit state has $S=0.5$ and $E=0$ [12]. The output state of the balanced entanglement concentrator has $E=1$ and $S=0$. Most importantly, the entanglement concentration has been made without throwing away any sub-ensemble of the initial two-qubit system.

A more general state, partially mixed-partially entangled, can be readily prepared by introducing the delay $\tau$ in one arm. If $\tau \neq 0$ and $\tau<|\delta t|$, where $\delta t$ is the twophoton coherence length, we obtain a state which is not completely entangled and not completely mixed. Such states are called Werner states [13] and lie between two points $(S, E)=(0.5,0)$ and $(S, E)=(0,1)$. Only recently, researchers have started to study these states experimentally in the cw domain [14. Such states are important in studying controllable decoherence in multi-qubit systems (for now, however, it is limited to two-qubit systems). Since our scheme offers readily controllable decoherence in the pulsed domain, we believe that it will be a useful tool to generate a multi-qubit entangled state and to study its decoherence in a controlled environment.

In conclusion, we have reported the experimental realization of entanglement concentration without local filtering and demonstrated a universal Bell-state synthesizer. Although typical type-II SPDC is used as a source, our Bell-state synthesizer is not affected by the pump source, the crystal properties, and the use of spectral filters. In addition, a more general state, partially mixedpartially entangled state can be readily generated. We believe that this new Bell-state synthesizer will be useful in experimental studies of quantum information science and as a building block of multi-particle entanglement. Finally, the entanglement concentration scheme based on dis-entanglement of decoherence causing and information carrying parts of the wavefunction may be applied to other quantum systems in which strong decoherence in one variable destroys entanglement in the other variable.

We would like to thank M.H. Rubin for helpful discussions. This research was supported by the U.S. Department of Energy, Office of Basic Energy Sciences, NSA, NSF, and ONR. The Oak Ridge National Laboratory is managed for the U.S. DOE by UT-Battelle, LLC, under contract No. DE-AC05-00OR22725.

[1] A. Einstein, B. Podolsky, and N. Rosen, Phys. Rev. 47, 777 (1935); J.S. Bell, Speakable and unspeakable in quantum mechanics, Cambridge University Press, New York, (1987).

[2] D.N. Klyshko and A.N. Penin, Sov. Phys.-Uspekhi 30, 716 (1987); A. Steane, Rep. Prog. Phys. 61, 117 (1998); J.P. Dowling, Phys. Rev. A 57, 4736 (1998).

[3] P.G. Kwiat et al., Phys. Rev. Lett. 75, 4337 (1995).

[4] Here we only consider experiments in which amplitude post-selection does not have to be assumed.

[5] T.E. Keller and M.H. Rubin, Phys. Rev. A 56, 1534 (1997); W.P. Grice and I.A. Walmsley, Phys. Rev. A 56, 1627 (1997).

[6] G. Di Giuseppe et al, Phys. Rev. A 56, R21 (1997);W. P. Grice et al, Phys. Rev. A 57, R2289 (1998); Y.-H. Kim et al., Phys. Rev. A 64, 011801(R) (2001); Y.-H. Kim et al., Phys. Rev. Lett. 86, 4710 (2001).

[7] R.T. Thew and W.J. Munro, Phys. Rev. A 63, 030302(R) (2001).

[8] Y.-H. Kim et al., Phys. Rev. A 62, 011802(R) (2000); ibid. 63, 060301(R) (2001); ibid. 63, 062301 (2001).

[9] Y.H. Shih and A.V. Sergienko, Phys. Lett. A 186, 29 (1994); Y.H. Shih and A.V. Sergienko, Phys. Lett. A 191, 201 (1994); M.H. Rubin, D.N. Klyshko, Y.H. Shih, and A.V. Sergienko, Phys. Rev. A 50, 5122 (1994).

[10] Our entanglement concentration scheme does not remove the intrinsic timing information present in type-II SPDC: it is simply made irrelevant to the quantum interference effect.

[11] Y.-H. Kim and W.P. Grice, to appear in J. Mod. Opt (2002); quant-ph/0207047.

[12] $S=-\sum_{i=1}^{4} \lambda_{i} \log _{4} \lambda_{i}$, where $\lambda_{i}$ are the eigenvalues of the density matrix $\rho . S=0.5$ because the initial state is a mixture of $\left|H_{1}\right\rangle\left|V_{2}\right\rangle$ and $\left|V_{1}\right\rangle\left|H_{2}\right\rangle$ of all four possible states.

[13] R.F. Werner, Phys. Rev. A 40, 4277 (1989).

[14] P.G. Kwiat et al., Nature 409, 1014 (2001); A.G. White et al., Phys. Rev. A 65, 012301 (2001). 